\title{
Treatment outcomes after adjuvant radiotherapy following surgery for patients with stage I endometrial cancer
}

\author{
Jiyoung Kim, MD1, Kyung-Ja Lee, MD, PhD¹, Kyung-Ran Park, MD, PhD¹, Boram Ha, MD², Yi-Jun Kim, MD', \\ Wonguen Jung, MD', Rena Lee, PhD', Seung Cheol Kim, MD, PhD ${ }^{3}$, Hye Sung Moon, MD, PhD², \\ Woong Ju, MD, PhD³, Yun Hwan Kim, MD, PhD³ , Jihae Lee, MD ${ }^{1}$ \\ ${ }^{1}$ Department of Radiation Oncology, Ewha Womans University School of Medicine, Seoul; \\ ${ }^{2}$ Proton Therapy Center, National Cancer Center, Goyang; \\ ${ }^{3}$ Department of Obstetrics and Gynecology, Ewha Womans University School of Medicine, Seoul, Korea
}

\begin{abstract}
Purpose: The purpose of this study is to evaluate the treatment outcomes of adjuvant radiotherapy using vaginal brachytherapy (VB) with a lower dose per fraction and/or external beam radiotherapy (EBRT) following surgery for patients with stage I endometrial carcinoma.

Materials and Methods: The subjects were 43 patients with the International Federation of Gynecology and Obstetrics (FIGO) stage I endometrial cancer who underwent adjuvant radiotherapy following surgery between March 2000 and April 2014. Of these, 25 received postoperative VB alone, while 18 received postoperative EBRT to the whole pelvis; 3 of these were treated with EBRT plus VB. The median EBRT dose was 50.0 Gy (45.0-50.4 Gy) and the VB dose was $24 \mathrm{~Gy}$ in 6 fractions. Tumor dose was prescribed at a depth of $5 \mathrm{~mm}$ from the cylinder surface and delivered twice per week.

Results: The median follow-up period for all patients was 57 months (range, 9 to 188 months). Five-year disease-free survival (DFS) and overall survival (OS) for all patients were $92.5 \%$ and $95.3 \%$, respectively. Adjuvant radiotherapy was performed according to risk factors and stage $I B$, grade 3 and lymphovascular invasion were observed more frequently in the EBRT group. Five-year DFS for EBRT and VB alone were $88.1 \%$ and $96.0 \%$, respectively $(p=0.42$ ), and 5 -year OS for EBRT and VB alone were $94.4 \%$ and $96 \%$, respectively $(p=0.38)$. There was no locoregional recurrence in any patient. Two patients who received EBRT and 1 patient who received VB alone developed distant metastatic disease. Two patients who received EBRT had severe complications, one each of grade 3 gastrointestinal complication and pelvic bone insufficiency fracture.

Conclusion: Adjuvant radiotherapy achieved high DFS and OS with acceptable toxicity in stage I endometrial cancer. VB (with a lower dose per fraction) may be a viable option for selected patients with early-stage endometrial cancer following surgery.
\end{abstract}

Keywords: Endometrial cancer, Intracavity radiotherapy, Conformal radiotherapy, Adjuvant radiotherapy

\section{Introduction}

Adenocarcinoma of the endometrium is the most common gynecologic malignancy in the United States. In Korea, the incidence of endometrial cancer has increased from 1.4 to 2.7 per 100,000 females between 1999 and 2012 [1]. Approximately $70 \%$ of endometrial cancers present as the International Federation of Gynecology and Obstetrics (FIGO) stage I disease, in which invasive neoplasm is confined to the uterus. The primary treatment for early-stage endometrial

Received 26 January 2016, Revised 8 June 2016, Accepted 30 June 2016.

Correspondence: Jihae Lee, MD, Department of Radiation Oncology, Ewha Womans University School of Medicine, 1071 Anyangcheon-ro, Yangcheon-gu, Seoul 07985, Korea. Tel: +82-2-2650-5331, Fax: +82-2-2654-0363, E-mail: jihae.leee@gmail.com

(c) This is an Open Access article distributed under the terms of the Creative Commons Attribution Non-Commercial License (http://creativecommons.org/ licenses/by-nc/4.0/) which permits unrestricted non-commercial use, distribution, and reproduction in any medium, provided the original work is properly cited.

www.e-roj.org 
cancer is generally hysterectomy and bilateral salpingooophorectomy with or without lymph node dissection. Patients with superficial myometrial invasion, grade 1 or 2, and which lack other high risk features such as lymphovascular invasion or older age, can be observed after hysterectomy. However adjuvant radiotherapy such as external beam radiotherapy (EBRT) and/or vaginal brachytherapy (VB) is performed for patients with intermediate or high risk stage I endometrial cancer to improve treatment outcome.

Previous randomized trials established that postoperative pelvic EBRT provides a significant improvement in local control, but this does not translate into a survival advantage and is associated with an increased incidence of side effects [2-4]. The most common site of local relapse in surgically staged patients is the vaginal vault. Randomized trials have shown that VB provides local control benefits almost equivalent to those of EBRT, but with less toxicity and better quality of life [2-6]. Although the postoperative treatment of FIGO stage I endometrial carcinoma has been a topic of vigorous discussion, a clear consensus has not been reached regarding treatment guidelines [7].

The aim of this study is to analyze the survival outcomes and complication rates according to adjuvant radiotherapy methods, and to determine whether VB with a lower dose per fraction is adequate for selected patients with stage I endometrial cancer.

\section{Materials and Methods}

\section{Patient eligibility}

We reviewed the records of patients who were diagnosed with endometrial carcinoma and received radiotherapy between March 2000 and April 2014. There were 56 patients with FIGO stage I endometrial adenocarcinoma who underwent adjuvant radiotherapy following surgery. We excluded 3 patients with non-endometrioid histology (carcinosarcoma, clear cell carcinoma), 9 patients with follow up less than 6 months, and 1 patient with incomplete treatment. Ultimately, 43 patients were enrolled in this study.

\section{Treatment}

1) Surgery: All 43 patients underwent hysterectomy with bilateral salpingo-oophorectomy, which was performed as follows: radical hysterectomy in 8 patients (18.6\%) and total hysterectomy in 35 patients (81.4\%). Selective pelvic/paraaortic lymph node dissection was also performed, as follows: no dissection in 3 patients (7.0\%), bilateral pelvic lymph node dissection in 20 patients (46.5\%), and additional para-aortic lymph node dissection in 20 patients (46.5\%) (Table 1).

2) Radiotherapy: Adjuvant radiation therapy was performed following surgery, according to risk factors. At Ewha Womans University Mokdong Hospital, patients with stage IA grade 1 received no further treatment after surgery, and stage IA grade 2 patients with at least one risk factor for disease recurrence (e.g., age $>60$ years, lymphovascular invasion, lower uterine segment involvement) received VB alone. EBRT or VB was performed for stage IA grade 3 and stage IB grade 1 or 2, while EBRT and/or VB were performed for stage IB grade 3. Twenty-five patients received postoperative $V B$ alone, while 18 received postoperative EBRT to the whole pelvis; of these patients, 3 were treated with EBRT plus VB (Table 2).

EBRT: Contrast-enhanced computed tomography (CT) simulation was obtained for all patients. Oral contrast (250 $\mathrm{mL}$ ) was given 3 hours prior to scanning. Patients were placed in the prone position with both arms placed over the head, and a commercially available belly-board device was used to spare the small bowel [8].

The target volume included the proximal vaginal, paravaginal, and parametrial tissues as well as the common iliac, external iliac, and internal iliac nodal regions, and the presacral region. The upper border was defined as the L4-5 interspace, the inferior border as the lower margin of the obturator foramen, and the lateral borders as $1 \mathrm{~cm}$ beyond the wall of the bony pelvis.

Three-dimensional conformal radiation therapy was planned with Pinnacle system (ver. 8.0m; Philips Medical Systems, Cleveland, OH, USA) and delivered with $10 \mathrm{MV}$ photons by a linear accelerator (Primus; Siemens, Malvern, PA, USA) using the four-field box technique. Radiation prescription dose is described in Table 2.

Brachytherapy: Brachytherapy was delivered using a cylinder connected to a high dose rate remote afterloading system (microSelectron; Nucletron, Veenendaal, The Netherlands) with an iridium-192 source. The cylinder diameter ranged from 2.5 to $3 \mathrm{~cm}$. The doses at $5 \mathrm{~mm}$ depth within the vaginal mucosa, rectum and bladder were documented according to the ICRU-38 criteria [9]. The biologically effective dose (BED) for tumor and late-responding tissues were calculated using the linear quadratic model. The $\alpha / \beta$ ratio for tumor was assumed to be $10 \mathrm{~Gy}$ and for rectum and bladder was assumed to be $3 \mathrm{~Gy}$ [10]. For the low-dose fractionation VB regimen, the BED for tumor recalculated to an equivalent 2-Gy dose was $28 \mathrm{~Gy}$ at $5 \mathrm{~mm}$ from the surface of the cylinder. 
Details of the radiotherapy regimens are presented in Table 2.

\section{Follow-up and response assessment}

The first follow-up visit was scheduled at 2 weeks after the completion of radiotherapy. Follow-up visits were then scheduled every 3 months for the first 2 years, every 6 months until 5 years, and annually thereafter. A gynecologic examination including vaginal assessment was performed at every follow-up visit and a Papanicolaou smear was done every 6 months. Imaging examinations included an annual CT of the abdomen and pelvis, magnetic resonance imaging (MRI) of the pelvis, and positron emission tomography-computed

Table 1. Patient characteristics

\begin{tabular}{|c|c|c|c|c|}
\hline Variable & $\mathrm{EBRT}^{\mathrm{a})}(\mathrm{n}=18)$ & $\operatorname{VB}(n=25)$ & Total $(n=43)$ & p-value \\
\hline Age (yr) & $55(31-71)$ & $56(38-58)$ & $55(31-71)$ & 0.52 \\
\hline \multicolumn{5}{|l|}{ Comorbidity } \\
\hline Diabetes & $1(5.5)$ & $3(12.0)$ & $4(9.1)$ & 0.47 \\
\hline Hypertension & 7 (38.9) & $11(44.0)$ & $18(40.9)$ & 0.74 \\
\hline Surgery & & & & 0.61 \\
\hline Radical hysterectomy & $4(22.2)$ & $4(16.0)$ & 8 (18.6) & \\
\hline Total hysterectomy & $14(77.8)$ & $21(84.0)$ & $35(81.4)$ & \\
\hline Lymph node dissection & & & & 0.066 \\
\hline None & $3(16.7)$ & $0(0)$ & $3(7.0)$ & \\
\hline BPLND & $10(55.5)$ & $10(40.0)$ & $20(46.5)$ & \\
\hline BPLND + PALND & $5(27.8)$ & $15(60.0)$ & $20(46.5)$ & \\
\hline FIGO stage & & & & 0.014 \\
\hline $\mathrm{IA}$ & $4(22.2)$ & $15(60.0)$ & $19(44.2)$ & \\
\hline IB & $14(77.8)$ & $10(40.0)$ & $24(55.8)$ & \\
\hline Grade & & & & 0.013 \\
\hline 1 & $4(22.2)$ & $5(20.0)$ & $9(20.9)$ & \\
\hline 2 & $5(27.8)$ & $17(68.0)$ & $22(51.2)$ & \\
\hline 3 & $9(50.0)$ & $3(12.0)$ & $12(27.9)$ & \\
\hline Lymphovascular invasion & & & & 0.013 \\
\hline Yes & $8(44.4)$ & $4(16.0)$ & $12(27.9)$ & \\
\hline No & 7 (38.9) & $21(84.0)$ & $28(65.1)$ & \\
\hline Unknown & $3(16.7)$ & $0(0)$ & $3(7.0)$ & \\
\hline Lower segment involvement & & & & 0.38 \\
\hline Yes & $3(16.7)$ & $2(8.0)$ & $5(11.6)$ & \\
\hline No & $15(83.3)$ & $23(92.0)$ & $38(88.4)$ & \\
\hline
\end{tabular}

Values are presented as median (range) or number (\%).

EBRT, external beam radiotherapy; VB, vaginal brachytherapy; BPLND, bilateral pelvic lymph node dissection; PALND, para-aortic lymph node dissection; FIGO, International Federation of Gynecology and Obstetrics.

a) Three patients in EBRT group were treated with EBRT plus VB.

Table 2. Treatment characteristics

\begin{tabular}{|c|c|c|c|c|c|}
\hline & \multicolumn{2}{|c|}{ Total dose } & \multirow{2}{*}{$\begin{array}{c}\text { EOD2 } \\
\text { (at } 0.5 \mathrm{~cm} \text { ) }\end{array}$} & \multicolumn{2}{|c|}{ Equivalent dose (BED3) } \\
\hline & EBRT & VB (at $0.5 \mathrm{~cm})$ & & Rectum & Bladder \\
\hline EBRT & $1.8 \mathrm{~Gy} \times 28 \mathrm{Fx}$ & 0 & 49.6 & 80.6 & 80.6 \\
\hline VB & 0 & $4 \mathrm{~Gy} \times 6 \mathrm{Fx}^{\mathrm{a})}$ & 28 & $65.4(37.5-106.8)$ & $41.8(16.5-57.8)$ \\
\hline $\mathrm{EBRT}+\mathrm{VB}$ & $\begin{array}{c}1.8 \mathrm{~Gy} \times 25-28 \mathrm{Fx} \\
(\mathrm{MB} 41.4 \mathrm{~Gy})\end{array}$ & $3 \mathrm{~Gy} \times 5-6 \mathrm{Fx}^{\mathrm{a})}$ & $57.4(54.9-60.2)$ & $99.4(89.1-111.5)$ & $95.7(87.7-108.3)$ \\
\hline
\end{tabular}

EBRT, external beam radiotherapy; VB, vaginal brachytherapy; EQD2, equivalent dose in 2 Gy fractions; BED3, biological effective dose calculated using $\alpha / \beta$ ratios of $3 \mathrm{~Gy}$; Fx, fraction; MB, midline block.

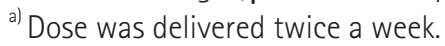


tomography (PET-CT).

Adverse effects were assessed by the Common Terminology Criteria for Adverse Events (CTCAE) ver. 3.0 criteria for gastrointestinal (GI), genitourinary, musculoskeletal and lymphatic side effects. Toxicities of grade 3 or greater were evaluated.

\section{Statistical analysis}

Kaplan-Meier analysis was used to calculate the rates of disease-free survival (DFS) and overall survival (OS), and a log-rank test was performed to compare the survival curves. Fisher's exact test was used to identify statistically significant difference in the incidence in toxicity between the EBRT and VB groups.

The Cox proportional hazard regression model was used for multivariate analysis. All statistical analyses were conducted with SPSS ver. 18.0 (SPSS Inc., Chicago, IL, USA) and p-values of $\leq 0.05$ were considered significant.

\section{Results}

\section{Patient and treatment characteristics}

The median age at the time of diagnosis was 55 years (range, 31 to 71 years). According to the FIGO 2009 system, 19 patients (44.2\%) had stage IA and 24 (55.8\%) had stage IB

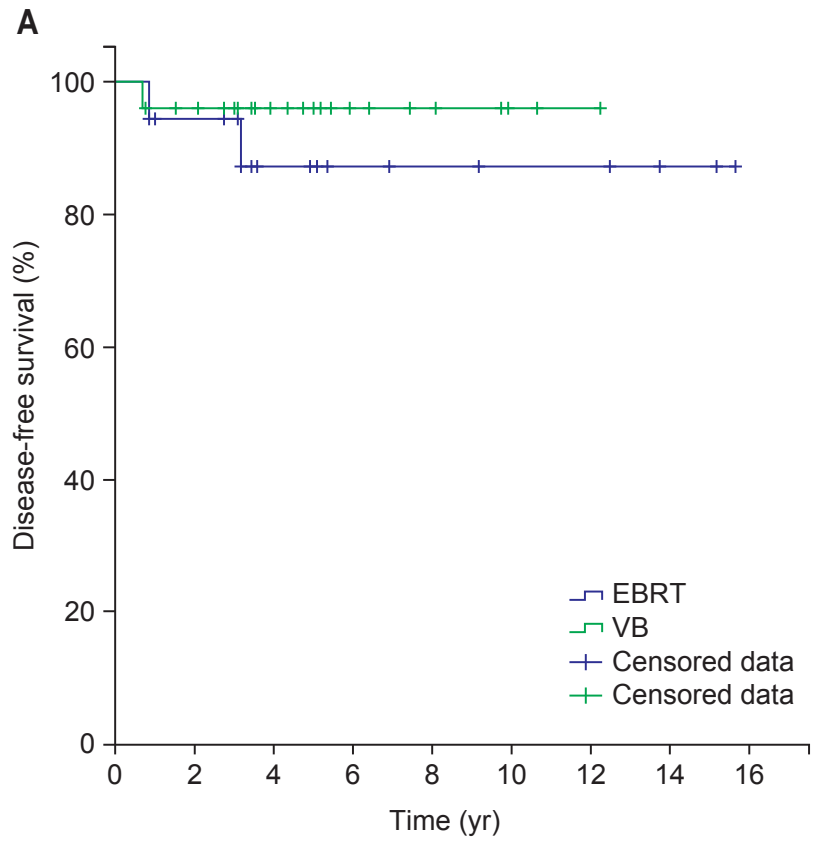

disease. Grade 1 was noted in 9 patients (20.9\%), grade 2 in 22 patients (51.2\%), and grade 3 in 12 patients (27.9\%). Lymphovascular invasion was identified in 12 patients (27.9\%) and lower uterine segment involvement was found in 5 patients (11.6\%). Stage IB, grade 3, and Iymphovascular invasion were more common in the EBRT group than the VB group (Table 1).

\section{Survival}

The median follow-up period of all patients was 57 months (range, 9 to 188 months). Death has occurred in 3 patients. Five-year DFS and OS for all patients were 92.5\% and 95.3\%, respectively. Five-year DFS for EBRT and VB alone were 88.1\% and $96.0 \%$, respectively ( $p=0.42$ ), and 5 -year OS for EBRT and VB alone were $94.4 \%$ and $96.0 \%$, respectively ( $p=0.38$ ). Kaplan-Meier curves showed no difference in 5-year DFS or OS according to radiation treatment group (Fig. 1).

\section{Failure patterns}

There was no locoregional recurrence in any patient, but 2 patients who received EBRT and 1 patient who received VB alone developed distant metastatic disease. The first of these 3 patients was 70 years old with FIGO stage IB, grade 3, and lymphovascular invasion. She developed metastases in the bone, lung, and adrenal gland. She was treated with salvage

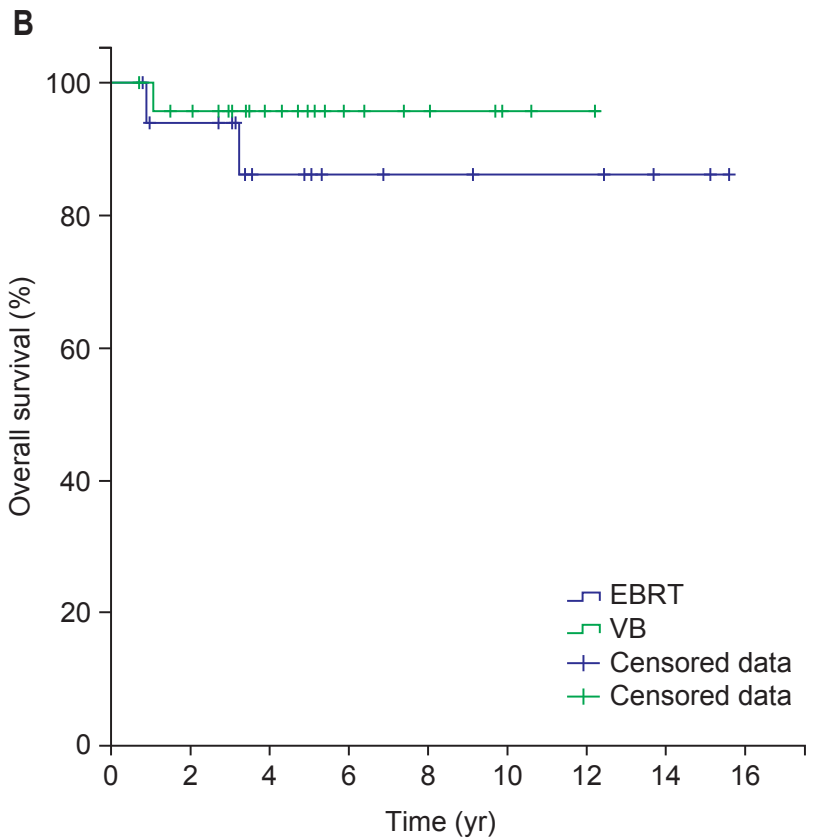

Fig. 1. Kaplan-Meier disease-free survival (A) and overall survival (B) curves. EBRT, external beam radiotherapy; VB, vaginal brachytherapy. 
surgery followed by palliative radiotherapy to the bone. The second patient was 55 years old with FIGO stage IB, grade 3 , and lymphovascular invasion and lung metastases were treated with chemotherapy. The third patient was 41 years old with FIGO stage IB, grade 2, and no Iymphovascular invasion. She developed multiple metastases (lung, kidney, liver, and brain) and was subsequently treated with palliative radiotherapy to the brain. All of these patients died as a result of metastatic disease.

\section{Complications}

The reported toxicities are summarized in Table 3. The incidence of acute grade 1 and 2 GI complications was significantly higher in the EBRT group. GI symptoms consisted of abdominal pain, frequency of bowel movements, or episodes of diarrhea and most genitourinary symptoms were reduced bladder capacity with urgency. Moderate to severe late complications ( $\geq$ grade 3 ) occurred in 2 patients, both 3 patients who were treated with EBRT plus VB had no severe complications. One patient who had no specific risk factor such as prior operation history was hospitalized with small bowel obstruction. Seventy-year old women with osteoporosis developed pelvic bone insufficiency fracture that responded to hospitalization and bed rest. Chronic lower extremity lymphedema was the most common lymphatic toxicity, occurring in 5 patients (28\%)

Table 3. Toxicity

\begin{tabular}{|c|c|c|c|}
\hline Side effect & $\begin{array}{c}\text { EBRT } \\
(n=18)\end{array}$ & $\begin{array}{c}\text { VB } \\
(n=25)\end{array}$ & p-value \\
\hline \multicolumn{4}{|l|}{ Acute side effect } \\
\hline \multicolumn{4}{|l|}{ Genitourinary } \\
\hline Any & $6(33.3)$ & $5(20.0)$ & 0.48 \\
\hline Severe ( $\geq$ grade 3 ) & $0(0)$ & $0(0)$ & - \\
\hline \multicolumn{4}{|l|}{ Gastrointestinal } \\
\hline Any & $12(66.7)$ & $0(0)$ & $<0.001$ \\
\hline Severe ( $\geq$ grade 3 ) & $0(0)$ & $0(0)$ & - \\
\hline \multicolumn{4}{|l|}{ Late } \\
\hline \multicolumn{4}{|l|}{ Gastrointestinal } \\
\hline Any & $1(5.6)$ & $0(0)$ & 0.42 \\
\hline Severe ( $\geq$ grade 3 ) & $1(5.6)$ & $0(0)$ & 0.42 \\
\hline \multicolumn{4}{|l|}{ Musculoskeletal } \\
\hline Any & $1(5.6)$ & $0(0)$ & 0.42 \\
\hline Severe ( $\geq$ grade 3 ) & $1(5.6)$ & $0(0)$ & 0.42 \\
\hline \multicolumn{4}{|l|}{ Lymphatics } \\
\hline Any & $5(27.8)$ & $2(20.0)$ & 0.11 \\
\hline Severe ( $\geq$ grade 3 ) & $0(0)$ & $0(0)$ & - \\
\hline
\end{tabular}

Values are presented as number (\%).

EBRT, external beam radiotherapy; $V B$, vaginal brachytherapy. in the EBRT group and 2 patients (20\%) in the VB group. Three patients in EBRT group underwent bilateral pelvic lymph node dissection and 2 patients in each group performed additional para-aortic lymph node dissection.

\section{Discussion and Conclusion}

In this study, we analyzed the outcomes of adjuvant radiotherapy for stage I endometrial cancer. VB with a low dose per fraction (4 Gy per fraction) yields high DFS and OS with low complication rates for selected patients with stage I endometrial cancer after surgery.

Several randomized trials have compared EBRT with observation and significant improvement of local control was observed in the EBRT group [2,3]. In consideration of no benefit of OS with EBRT and of the fact that the most common site of recurrence was vagina, comparing VB with EBRT have been performed in numerous prospective and retrospective studies (Table 4) $[4-6,11,12]$. In the Post-Operative Radiation Therapy in Endometrial Carcinoma (PORTEC)-2 trial [6], there was no statistically significant difference in the 5 -year vaginal recurrence (1.8\% vs. 1.6\%) between the VB and EBRT groups. There was no significant difference in OS, and GI toxicity was more frequent in the EBRT group. This trial demonstrated that VB alone offers tolerable vaginal control, with lower morbidity than EBRT. In 2012, an updated Cochrane systematic review was published [13]. The authors concluded that EBRT lowers locoregional recurrence but does not impact OS with significant toxicities. It is noteworthy that there has recently been a significant increase in the use of postoperative VB alone to treat endometrial cancer [14]. But in previous trials, patients have been subdivided in various ways according to the prognostic features including age, tumor grade, myometrial invasion, and lymphovascular invasion and it is therefore important to perform adjuvant radiotherapy according to these risk factors [15].

In the present study, patients with risk factors such as stage IB, grade 3 and Iymphovascular invasion were more frequently observed in the EBRT group. Both 5-year DFS and OS in patients treated with VB alone were 96.0\%, and there was no difference in 5-year DFS or OS between the EBRT and VB alone groups. Locoregional recurrence did not occur and 3 patients ( 2 who received EBRT and 1 who received VB alone) died due to distant recurrence. Toxicity profile of $V B$ group is more favorable than EBRT group. Acute GI toxicity was significantly higher in EBRT group compared with VB group and there was no $\geq$ grade 3 late toxicity in VB group, while 2 


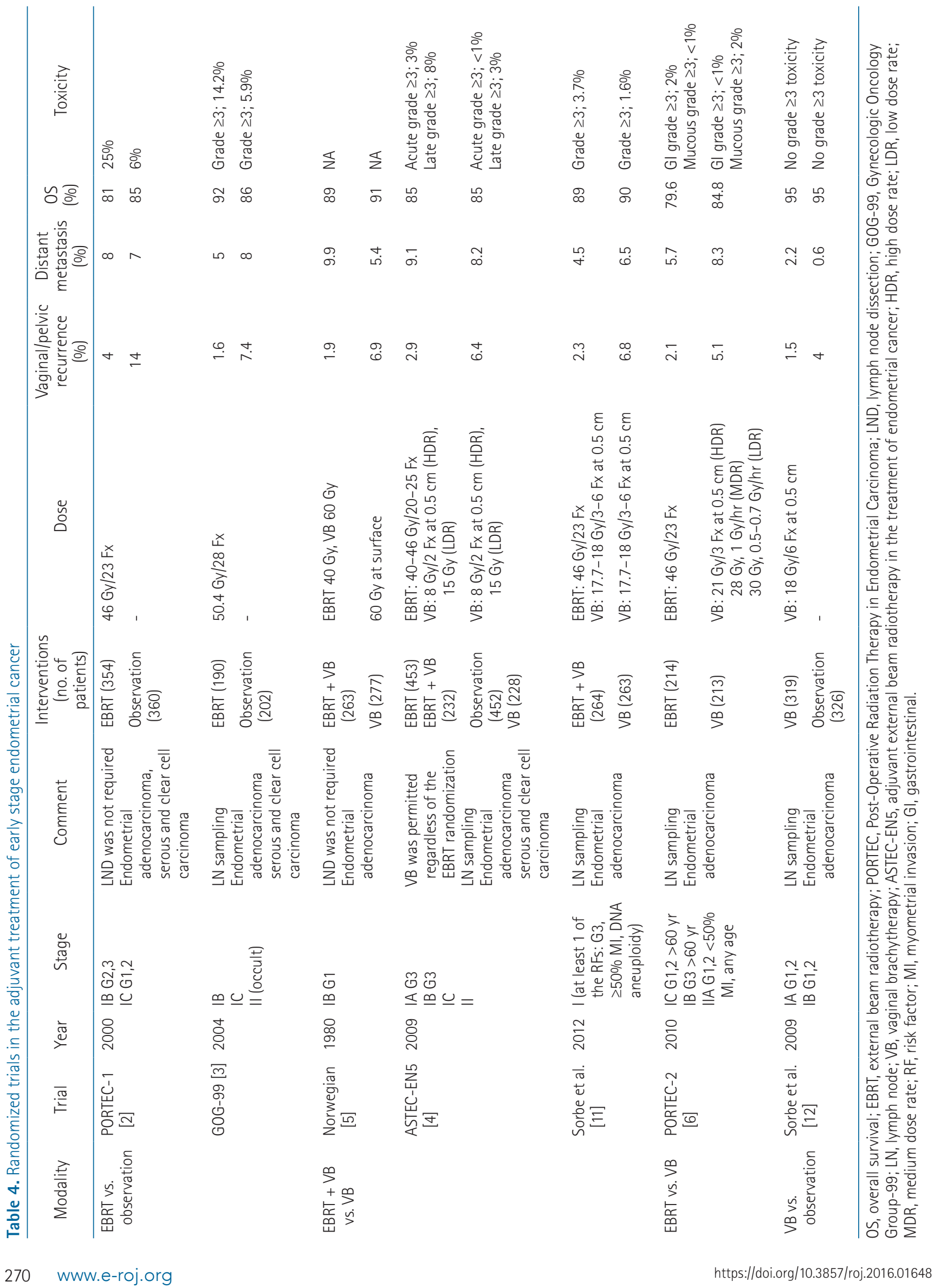


patients in our EBRT group reported severe late complications (small bowel obstruction and pelvic bone insufficiency fracture). This favorable result is comparable with those of the previous studies which include VB alone group in early-stage endometrial cancer patients [16-20].

The VB dose in numerous retrospective studies of stage I endometrial cancer ranged from 15 to 36 Gy in 2-6 fractions, and the most common fractionation regimen was $7 \mathrm{~Gy} \times$ 3 fractions prescribed at a depth of $5 \mathrm{~mm}$, as used in the PORTEC-2 trial $[2,6]$. Townamchai et al. [21] reported that lower dose regimens ( $4 \mathrm{~Gy} \times 6$ fractions prescribed to the cylinder surface) showed excellent locoregional control with minimal morbidity. Although many studies have evaluated the optimal fraction size and total dose of $V B$, these factors have not been defined.

In 2005, Sorbe et al. [22] compared a regimen of $2.5 \mathrm{~Gy}$ $\times 6$ fractions with that of $5 \mathrm{~Gy} \times 6$ fractions and reported similar treatment efficacy; however, the higher dose regimen showed statistically significant increases in vaginal toxicities such as vaginal shortening, mucosal atrophy, and bleeding. In our study, we performed VB with a lower dose per fraction ( 4 Gy $\times 6$ fractions) and the goal of lowering fraction size is to maintain a similar tumor control rate, while reducing complications.

The limitations of this study are the small sample size and the underlying disadvantages associated with the retrospective study design. Patients in the EBRT group generally had more advanced disease, which could have introduced selection bias. The follow-up and toxicity data could have been underreported by physicians, and we did not record data of post-VB vaginal toxicity, which is the most common complication after brachytherapy. Further studies with a prospective study design are necessary to confirm our findings.

In conclusion, VB with a regimen of 24 Gy in 6 fractions showed no locoregional recurrence, no differences in 5-year DFS and OS compared with the EBRT group but minimal late morbidity. This regimen may be a viable option for selected patients with stage I endometrial cancer.

\section{Conflict of Interest}

No potential conflict of interest relevant to this article was reported.

\section{References}

1. Jung KW, Won YJ, Kong HJ, et al. Cancer statistics in Korea: incidence, mortality, survival, and prevalence in 2012. Cancer Res Treat 2015;47:127-41.

2. Creutzberg $C L$, van Putten $W L$, Koper PC, et al. Surgery and postoperative radiotherapy versus surgery alone for patients with stage- 1 endometrial carcinoma: multicentre randomised trial. Lancet 2000;355:1404-11.

3. Keys HM, Roberts JA, Brunetto VL, et al. A phase III trial of surgery with or without adjunctive external pelvic radiation therapy in intermediate risk endometrial adenocarcinoma: a Gynecologic Oncology Group study. Gynecol Oncol 2004:92:744-51.

4. ASTEC/EN.5 Study Group, Blake P, Swart AM, et al. Adjuvant external beam radiotherapy in the treatment of endometrial cancer (MRC ASTEC and NCIC CTG EN.5 randomised trials): pooled trial results, systematic review, and meta-analysis. Lancet 2009;373:137-46.

5. Aalders J, Abeler V, Kolstad P, Onsrud M. Postoperative external irradiation and prognostic parameters in stage I endometrial carcinoma: clinical and histopathologic study of 540 patients. Obstet Gynecol 1980;56:419-27.

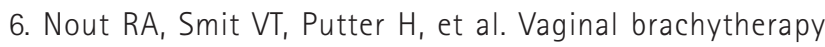
versus pelvic external beam radiotherapy for patients with endometrial cancer of high-intermediate risk (PORTEC-2): an open-label, non-inferiority, randomised trial. Lancet 2010;375:816-23.

7. Klopp A, Smith BD, Alektiar K, et al. The role of postoperative radiation therapy for endometrial cancer: executive summary of an American Society for Radiation Oncology evidencebased guideline. Pract Radiat Oncol 2014;4:137-44.

8. Martin J, Fitzpatrick K, Horan G, et al. Treatment with a belly-board device significantly reduces the volume of small bowel irradiated and results in low acute toxicity in adjuvant radiotherapy for gynecologic cancer: results of a prospective study. Radiother Oncol 2005;74:267-74.

9. Nag S, Erickson B, Parikh S, Gupta N, Varia M, Glasgow G. The American Brachytherapy Society recommendations for highdose-rate brachytherapy for carcinoma of the endometrium. Int J Radiat Oncol Biol Phys 2000;48:779-90.

10. Fowler JF. 21 years of biologically effective dose. Br J Radiol 2010;83:554-68.

11. Sorbe $B$, Horvath $G$, Andersson $H$, Boman $K$, Lundgren $C$, Pettersson B. External pelvic and vaginal irradiation versus vaginal irradiation alone as postoperative therapy in mediumrisk endometrial carcinoma: a prospective randomized study. Int J Radiat Oncol Biol Phys 2012;82:1249-55.

12. Sorbe B, Nordstrom B, Maenpaa J, et al. Intravaginal brachytherapy in FIGO stage I low-risk endometrial cancer: a controlled randomized study. Int J Gynecol Cancer 2009;19:873-8.

13. Kong A, Johnson N, Kitchener HC, Lawrie TA. Adjuvant radiotherapy for stage I endometrial cancer: an updated 
Cochrane systematic review and meta-analysis. J Natl Cancer Inst 2012;104:1625-34.

14. Meyer LA, Bohlke K, Powell MA, et al. Postoperative radiation therapy for endometrial cancer: American Society of Clinical Oncology Clinical Practice Guideline Endorsement of the American Society for Radiation Oncology Evidence-Based Guideline. J Clin Oncol 2015;33:2908-13.

15. DiSaia PJ, Creasman WT, Boronow RC, Blessing JA. Risk factors and recurrent patterns in Stage I endometrial cancer. Am J Obstet Gynecol 1985;151:1009-15.

16. Chadha M, Nanavati PJ, Liu P, Fanning J, Jacobs A. Patterns of failure in endometrial carcinoma stage IB grade 3 and IC patients treated with postoperative vaginal vault brachytherapy. Gynecol Oncol 1999;75:103-7.

17. Anderson JM, Stea B, Hallum AV, Rogoff E, Childers J. Highdose-rate postoperative vaginal cuff irradiation alone for stage IB and IC endometrial cancer. Int J Radiat Oncol Biol Phys 2000;46:417-25.

18. Solhjem MC, Petersen IA, Haddock MG. Vaginal brachytherapy alone is sufficient adjuvant treatment of surgical stage
I endometrial cancer. Int J Radiat Oncol Biol Phys 2005;62:1379-84.

19. Atahan IL, Ozyar E, Yildiz F, et al. Vaginal high dose rate brachytherapy alone in patients with intermediate- to highrisk stage I endometrial carcinoma after radical surgery. Int J Gynecol Cancer 2008;18:1294-9.

20. Diavolitsis V, Rademaker A, Lurain J, Hoekstra A, Strauss J, Small W Jr. Clinical outcomes in international federation of gynecology and obstetrics stage IA endometrial cancer with myometrial invasion treated with or without postoperative vaginal brachytherapy. Int J Radiat Oncol Biol Phys 2012;84:415-9.

21. Townamchai $K$, Lee $L$, Viswanathan AN. A novel low dose fractionation regimen for adjuvant vaginal brachytherapy in early stage endometrioid endometrial cancer. Gynecol Oncol 2012;127:351-5.

22. Sorbe B, Straumits A, Karlsson L. Intravaginal high-dose-rate brachytherapy for stage I endometrial cancer: a randomized study of two dose-per-fraction levels. Int J Radiat Oncol Bio Phys 2005;62:1385-9. 